\title{
TERRESTRIAL SNAILS IN FLOOD DEPOSITS OF THE BESKID MAŁY RANGE (CARPATHIANS, SOUTHERN POLAND)
}

\author{
WiTOLD PAWEŁ ALEXANDROWICZ
}

\begin{abstract}
Chair of General Geology and Geotourism, Faculty of Geology, Geophysics and Environment Protection, AGH University of Science and Technology, al. Mickiewicza 30, 30-059 Cracow, Poland (e-mail: wpalex@geol.agh.edu.pl); (1) https://orcid.org/0000-0002-5403-6696
\end{abstract}

\begin{abstract}
Long-term research on the malacofauna of the Beskid Mały, one of the ranges of the Western Beskidy Mts, was based on flood deposits of the streams cutting through the massif. Samples from limestone areas (called Andrychów Klippes) and those from the Żurawnica massif complemented the material. The total of ca. 400 samples yielded 78 species of terrestrial snails. The malacofauna was characterised in terms of its ecological and zoogeographical composition. The material provided the basis for determining the zones of occurrence and distribution of the component taxa within the Beskid Mały range. The ecological, zoogeographical and species diversity of the assemblages made it possible to distinguish four types of fauna, each connected with certain parts of the Beskid Mały range, which differ in their geological structure, relief and the degree of anthropogenic transformations.
\end{abstract}

KEY WORDS: terrestrial snails, biodiversity, Beskid Mały, Carpathians, southern Poland

\section{INTRODUCTION}

Research on the malacofauna of the Polish Carpathians started in the late 19th century. Since then numerous papers on mollusc fauna inhabiting parts of this mountain range have been published (STOBIECKI 1880, 1883, KOTULA 1884, BĄKOWSKI 1883, 1884, URBAŃSKI 1932, 1939, 1962, DYDUCH-FALNIOWSKA 1991, STWORZEWICZ 2000, SULIKOWSKA-DROZD 2002, ALEXANDROWICZ 2003, 2011, SULIKOWSKADROZD \& HORSÁK 2007, KASZUBA \& STWORZEWICZ 2018 and many others). Papers on the malacofauna of certain vegetation formations have also been published (DZIĘCZKOWSKI 1971, 1972, 1988), as well as on molluscs living in anthropogenically transformed areas, for example ruined castles (ALEXANDROWICZ 1995, 2013a). In this context the malacofauna of the Beskid Mały has been very little studied. The main purpose of this paper is to characterise the range's mollusc fauna and to estimate the species diversity and distribution in its various parts.

The major part of the material was obtained from flood deposits which accumulate on valley floors and floodplains during high water levels. Their characteristics depend on a number of factors, for example the shape and course of the river or stream bed, width, height and form of floodplain. Other factors include the presence of natural and/or anthropogenic barriers (bushes, tree trunks, hydrotechnical objects, bridge pylons). The principal components of flood sediments are wood fragments and plant debris, fine sand and mud, as well anthropogenic waste. Mollusc shells are often present in such sediments (e.g. AlEXANDROWICZ 1997a, 2002, ČEJKA et al. 2008, MYŠÁK \& HORSÁK 2011, ČILIAK et al. 2015).

Mollusc shells are characterised by relatively poor resistance to mechanical impact, especially in high-energy environments. As a result, during floods shells are transported only for relatively short distances (e.g. AlEXANDROWICZ 2000a, b, c, IlG et al. 2009, AlEXANDROWICZ \& ALEXANDROWICZ 2011). Shell accumulations in sediments (thanatocoenosis; WASMUND 1926) can be indigenous or paraindigenous (allocoenosis or even necrocoenosis; 
ALEXANDrowicz \& AleXANDrowicz 2011). Thus, they are limited to relatively small areas and their species composition is very similar to that of living malacocoenoses of the area adjacent to the sediment deposition. This method has been successfully used in faunistic studies (e.g. AlEXANDrowicz 1997a, 2000a, b, c, 2002, ČEJKA et al. 2008, ILG et al. 2009), in particular for qualitative analyses, while determining whether particular species are present in the area.

\section{STUDY AREA}

The Beskid Mały is a part of the Western Beskidy Mountain range (Fig. 1A). It is a small range extending for $35 \mathrm{~km}$ in $\mathrm{W}-\mathrm{E}$ direction and $15 \mathrm{~km}$ in $\mathrm{N}-\mathrm{S}$ direction. The Beskid Mały range is divided into two uneven parts by the meridionally running Soła River valley. The smaller western part culminates in the highest summit of the range - Czupel $(933 \mathrm{~m}$ a.s.l.). The malacological studies were conducted in the eastern part of the Beskid Mały range (Fig. 1B). It consists of three main ridges converging in the Leskowiec massif (922 $\mathrm{m}$ a.s.l.). The study area is built of Cretaceous and Palaeogene flysch formations assigned to the Silesian, sub-Silesian, and Magura units represented by sandstones and shales, with a smaller proportion of conglomerates. The northern part is characterised by the presence of formations with the prevalent shales assigned to the Silesian and Sub-Silesian units. The most important lithological units in the region are lower Cretaceous beds: Wierzowa and Lgota belonging to the Silesian Unit (KSIĄŻKIEWICZ 1932, 1951, PAUL et al. 1996). In the middle and southern parts upper-Cretaceous and Palaeocene thick-bedded sandstones and conglomerates (Godula and Istebna beds) of the Silesian Unit are predominant (KSIĄŻKIEWICZ 1932, 1951, PAUL et al. 1996). In the region of the town

of Sucha Beskidzka thick-bedded, slightly calcareous sandstones and conglomerates appear. These are the Ciężkowice beds of the Magura Unit. They form the small range of Żurawnica (ALEXANDROWICZ \& ALEXANDROWICZ 2006, CIESZKOWSKI et al. 2006) (Fig. 1B). The lithological features of the bedrock determine the morphology of the area. Its northern part is characterised by a moderately hilly relief and wide, flat-bottomed valleys. The area is mostly deforested. In the zone of the Godula and Istebna beds steep hills are overgrown by solid forest complexes, chiefly spruce-beech-fir forests with patches of Carpathian beech forest (Dentario glandulosae-Fagetum). The massif is cut through by narrow valleys with relatively steep slopes. Farmland and pastures cover only the lower parts of the slopes. In the last 2-3 decades the farming activities in the Beskid Mały range have been greatly limited and formerly cultivated fields have been taken over by scrub communities and young forests.

In the northern part of the study area, in the environs of the town of Andrychów, there are four isolated spots with carbonate rock: the upper-Jurassic, upper-Cretaceous and Palaeogene limestones and marls representing the Andrychów series (KSIĄŻKIEWICZ 1932, 1951, GASIŃSKI 1998) (Fig. 1B).

\section{MATERIAL AND METHODS}

The material was obtained mostly from flood deposits accumulated in stream valleys during summer floods and short-term storm high water levels. The material was collected for over a decade, starting in 2001. More than 250 samples of the sediments were taken in 11 valleys: Choczenka $(\mathrm{CH})$, Ponikiewka (PO), Rzyczanka (RZ), Targaniczanka (TR), Wielka Puszcza (WP), Kocierzanka (KO), Stryszawka (ST), Tarnawka (TA), Jaszczurówka (JA), Bulówka (BU), and Pilny Potok (PP) (Fig. 1B), following the methods specified by ALEXANDROWICZ \& ALEXANDROWICZ (2011). The material obtained after the flood of July 2001 (35 samples) was dealt with in an earlier paper (ALEXANDROWICZ 2002). Additional samples were taken in the area of carbonate outcrops near Andrychów with the use of $5 \times 5 \mathrm{~cm}$ square frame (DZIĘCZKOWSKI 1972, 1988). The research was conducted since the 1990s, initially at the locality of

Pańska Góra (Pg) and then also at nearby limestone quarries (Roczyny (Ro), Targanice (Tg), and Inwałd (In)) (Fig. 1B). The total number of samples from the area called Andrychów Klippes was 120; 30 of them were selected as the basis for an earlier paper (ALEXANDROWICZ 1994). The materials were supplemented by the results of malacofaunal analyses from the range of Żurawnica (ALEXANDROWICZ \& ALEXANDROWICZ 2006) (Fig. 1B) and by distributional data from the literature (RIEDEL 1998, WIKTOR 2004).

A total of ca. 400 samples provided the basis for research. The samples were dried and then complete mollusc shells and identifiable fragments were included in the analysis. The identification was based on guides (KERNEY et al. 1983, WIKTOR 2004, WELTER-SCHULTES 2012, HORSÁK et al. 2013) and comparative collections. Slugs (Agriolimacidae, 


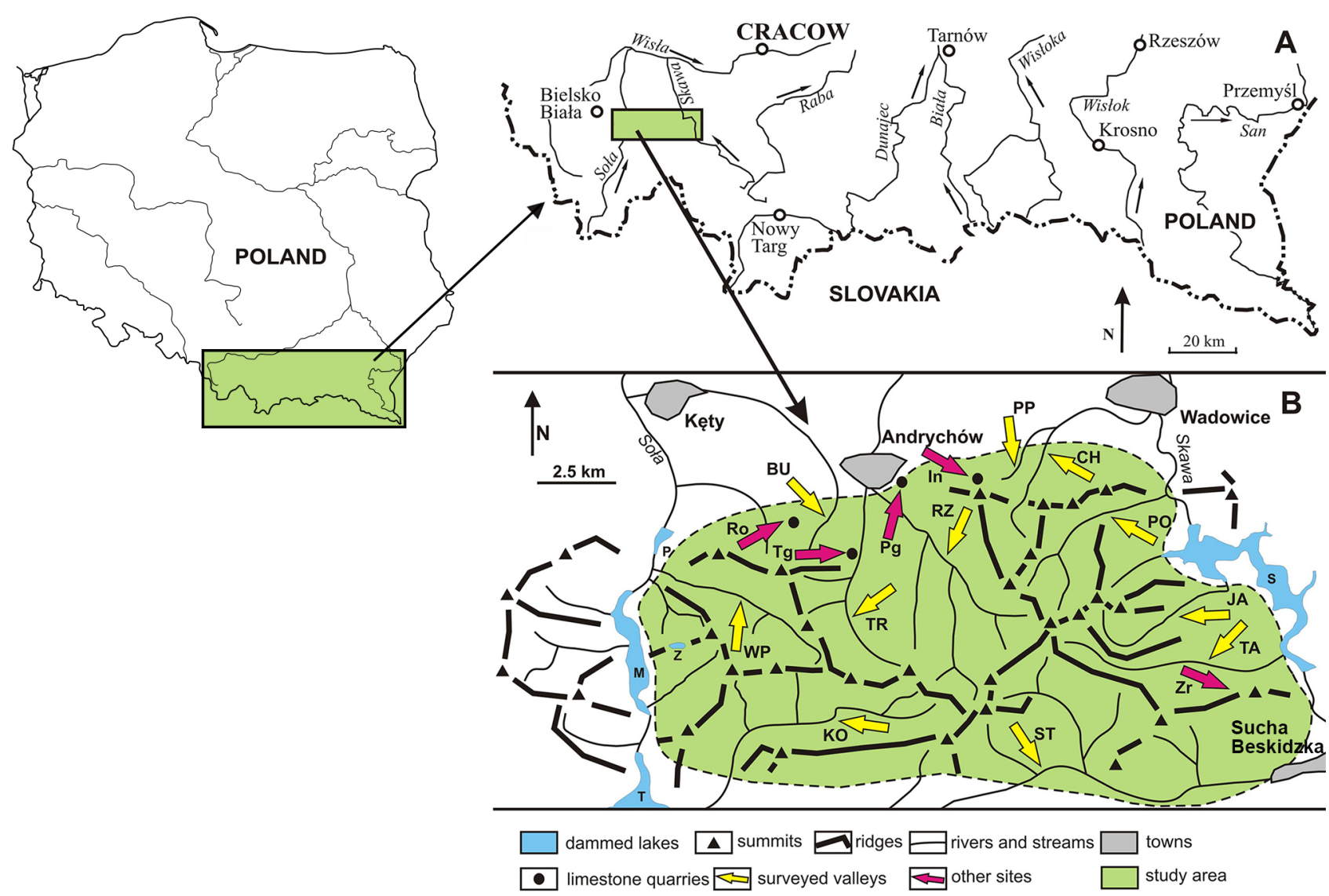

Fig. 1. Location of the study area (A), and sampling sites (B). Dam reservoirs: P - Porąbka, M - Międzybrodzie Żywieckie, T - Tresna, S - Świnna Poręba, Z - Żar; Valleys: BU - Bulówka, CH - Choczenka, JA - Jaszczurówka, KO - Kocierzanka, PO - Ponikiewka, PP - Pilny Potok, RZ - Rzyczanka, ST - Stryszawka, TA - Tarnawka, TR - Targaniczanka, WP Wielka Puszcza; Other sampling sites: In - Inwałd, Ro - Roczyny, Pg - Pańska Góra, Tg - Targanice, Zr - Żurawnica

Arionidae, Boettgerillidae, Limacidae and Milacidae), freshwater snails and bivalves were excluded from the analysis. The classification into ecological groups was based on schemes developed by LOŽEK (1964), ALEXANDROWICZ \& ALEXANDROWICZ (2011) and JUŘIČKOVÁ et al. (2014), the zoogeographical division followed ALEXANDROWICZ \& ALEXANDROWICZ
(2011). The constancy analysis (ALEXANDROWICZ \& ALEXANDROWICZ 2011) distinguished commonly occurring species, as well as taxa which are rare in the area. The use of similarity dendrogram (MORISITA 1959) made it possible to identify four types of fauna. The statistical analysis was carried out using the PAST software package (HAMMER et al. 2001).

\section{RESULTS AND DISCUSSION}

The mollusc fauna of the eastern part of the Beskid Mały range is rich and diverse. It includes 78 species of terrestrial snails, representing 22 families presently living in the Beskid Mały, and three taxa which have gone extinct in the area during the last several decades (Table 1). Compared with other ranges of the Flysch Carpathians, the malacofauna shows a similar species diversity: Babia Góra massif - 78 species (ALEXANDROWICZ 2003, 2011), Gorce 53 species (KASZUBA \& STWORZEWICZ 2018), Tatra Mts - 70 species (DYDUCH-FALNIOWSKA 1991). Richer and more diverse mollusc assemblages are known from the Pieniny Mts -84 species (URBAŃSKI 1939, Stworzewicz 2000), the Podhale Basin - 81 species (ALEXANDROWICZ 1997b, 2000b, c, 2013b) and Bieszczady and Beskid Niski Mts - 90 species (STWORZEWICZ \& PAWŁOWSKI 2000, SULIKOWSKADROZD 2002, SULIKOWSKA-DROZD \& HORSÁK 2007). Nevertheless, it should be emphasised that these areas are built mostly of carbonate rocks (limestones and/or dolomites - the Tatra Mts and the Pieniny Mts) or from strongly calcareous flysch formations (the Podhale Basin). The great content of carbonates in the bedrock favours mollusc diversity. In accordance with the data presented by RIEDEL (1988) 105 species of terrestrial shelled snails live in the Polish Carpathians. 
Table 1. Taxonomic composition of the malacofauna of the Beskid Mały. C-I - C-V (constancy classes after ALEXANDROWICZ \& AlEXANDROWICZ 2011). Ecological groups (after LOŽEK 1964, ALEXANDROWICZ \& ALEXANDROWICZ 2011, JUŘIČKOVÁ et al. 2014): $\mathrm{F}$ - shade-loving species: $\mathrm{F}_{\mathrm{F}}$ - shade-loving, forest species, $\mathrm{F}_{\mathrm{B}}$ - shade-loving species living in light forests and bushy zones, $\mathrm{F}_{\mathrm{H}}$ - shade-loving species of humid habitats; $\mathrm{O}$ - open-country species: $\mathrm{O}_{\mathrm{X}}$ - xerophilous and petrophilous species, $\mathrm{O}_{\mathrm{O}}$ - open-country species; $\mathrm{M}$ - mesophilous species: $\mathrm{M}_{\mathrm{D}}$ - mesophilous species of dry habitats, $M_{I}$ - mesophilous species of moderately wet habitats, $M_{H}-$ mesophilous species of wet habitats; $\mathrm{H}$ - hygrophilous species. Zoogeographical groups (after ALEXANDROWICZ \& ALEXANDROWICZ 2011): HL - widely distributed species: Hl - Holarctic species, Pl - Palaearctic species, Ep - European species, Es - Euro-Siberian species; ME - CentralEuropean species: $\mathrm{Me}$ - Central-European lowland and upland species, $\mathrm{Ma}$ - Central-European mountain species; EO - species of narrow distribution: Ee - East-European species, Eb - Pontic and Balkan species, Em - Mediterranean species, Ew - West-European species, Ba - Boreal-Alpine species

\begin{tabular}{|c|c|c|c|c|c|c|c|c|c|c|c|c|c|c|c|c|c|c|c|}
\hline 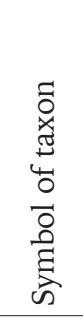 & 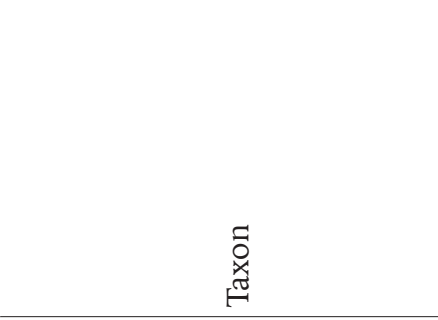 & 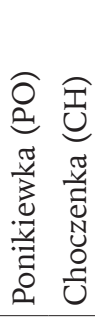 & 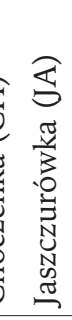 & 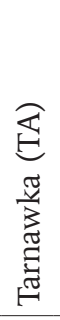 & 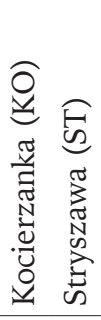 & 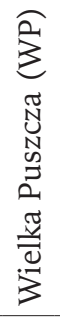 & 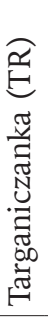 & 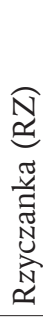 & 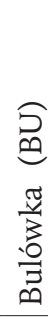 & 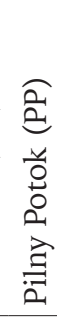 & $\begin{array}{l}\Xi \\
\Xi \\
0 \\
\frac{0}{\pi} \\
\Xi \\
\Xi\end{array}$ & 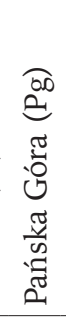 & 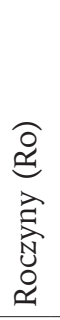 & 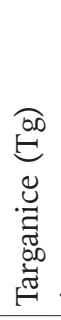 & 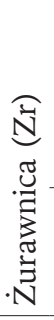 & 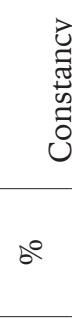 & $\frac{\mathscr{N}}{\stackrel{心}{U}}$ & 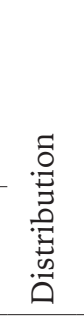 & 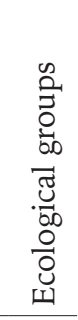 \\
\hline \multicolumn{20}{|c|}{ Aciculidae } \\
\hline Apa & Acicula parcelineata (Cless.) & & & & + & & & & & + & & & & & & 13 & I & $\mathrm{Ma}$ & $\mathrm{F}_{\mathrm{F}}$ \\
\hline Ppo & Platyla polita (Hartm.) & $+\quad+$ & + & + & $+\quad+$ & + & + & + & + & + & & & + & + & + & 87 & $\mathrm{~V}$ & $\mathrm{Me}$ & $\mathrm{F}_{\mathrm{F}}$ \\
\hline \multicolumn{20}{|c|}{ Ellobiidae } \\
\hline $\mathrm{Cmi}$ & Carychium minimum Müll. & + & + & + & $+\quad+$ & + & + & + & + & + & & & & & & 69 & IV & Es & $\mathrm{H}$ \\
\hline Ctr & Carychium tridentatum (Risso) & $+\quad+$ & + & + & $+\quad+$ & + & + & + & + & + & + & + & + & + & + & 100 & V & Ep & $\mathrm{M}_{\mathrm{H}}$ \\
\hline \multicolumn{20}{|c|}{ Succineidae } \\
\hline Spu & Succinea putris (L.) & ++ & + & + & ++ & + & + & + & + & + & & & & & & 69 & IV & Es & $\mathrm{H}$ \\
\hline Sob & Succinella oblonga (Drap.) & + & + & & $+\quad+$ & + & & + & + & + & & & & & & 50 & III & Es & $\mathrm{M}_{\mathrm{H}}$ \\
\hline \multicolumn{20}{|c|}{ Cochlicopidae } \\
\hline Clu & Cochlicopa lubrica (Müll.) & $+\quad+$ & + & + & $+\quad+$ & + & + & + & + & + & + & + & + & + & + & 100 & $\mathrm{~V}$ & $\mathrm{Hl}$ & $\mathrm{M}_{\mathrm{I}}$ \\
\hline $\mathrm{Clb}$ & Cochlicopa lubricella (Rossm.) & + & + & & + & & & & & & + & + & + & + & & 44 & III & $\mathrm{Hl}$ & $M_{D}$ \\
\hline \multicolumn{20}{|c|}{ Pupillidae } \\
\hline Pmu & Pupilla muscorum (L.) & & & & & & & & & & + & + & + & + & & 25 & II & $\mathrm{Hl}$ & $\mathrm{O}_{\mathrm{O}}$ \\
\hline Ppr & Pupilla cf. alpicola (Charp.) & & & & + & & & & & & & & & & & 6 & I & $\mathrm{Me}$ & $\mathrm{H}$ \\
\hline \multicolumn{20}{|c|}{ Valloniidae } \\
\hline Vco & Vallonia costata (Müll.) & $+\quad+$ & + & + & $+\quad+$ & & & + & + & + & & & & & + & 69 & IV & $\mathrm{Hl}$ & $\mathrm{O}_{\mathrm{O}}$ \\
\hline Vpu & Vallonia pulchella (Müll.) & $+\quad+$ & + & + & $+\quad+$ & + & + & + & + & & + & + & + & + & + & 94 & $\mathrm{~V}$ & $\mathrm{Hl}$ & $\mathrm{O}_{\mathrm{O}}$ \\
\hline Aac & Acanthinula aculeata (Müll.) & + & + & + & + & & & & + & + & & & & & + & 44 & II & $\mathrm{Pl}$ & $\mathrm{F}_{\mathrm{F}}$ \\
\hline \multicolumn{20}{|c|}{ Vertiginidae } \\
\hline Cas & Columella aspera Wald. & $+\quad+$ & + & + & $+\quad+$ & + & + & + & + & + & + & + & + & + & & 94 & $\mathrm{~V}$ & $\mathrm{Hl}$ & $M_{I}$ \\
\hline Ced & Columella edentula (Drap.) & ++ & + & + & $+\quad+$ & + & + & + & + & + & + & + & + & + & + & 100 & V & $\mathrm{Hl}$ & $\mathrm{F}_{\mathrm{H}}$ \\
\hline Tcr & Truncatellina cylindrica (Fér.) & & & & & & & & & & + & + & + & + & + & 31 & II & Ep & $\mathrm{O}_{\mathrm{O}}$ \\
\hline Val & Vertigo alpestris Ald. & + & + & & + & & & & & & & & & & + & 25 & II & $\mathrm{Ba}$ & $\mathrm{F}_{\mathrm{B}}$ \\
\hline Van & Vertigo angustior Jeffr. & & & & + & & & & & & & & & & & 6 & I & Ep & $\mathrm{M}_{\mathrm{H}}$ \\
\hline Vps & Vertigo pusilla Müll. & $+\quad+$ & + & + & $+\quad+$ & + & & & + & + & + & & & + & + & 75 & IV & Ep & $\mathrm{F}_{\mathrm{F}}$ \\
\hline Vpy & Vertigo pygmaea (Drap.) & $+\quad+$ & + & + & $+\quad+$ & + & + & + & + & + & + & + & + & + & & 94 & $\mathrm{~V}$ & $\mathrm{Hl}$ & $\mathrm{O}_{\mathrm{O}}$ \\
\hline Vsu & Vertigo substriata (Jeffr.) & & + & + & + & & & & & & & & & & & 19 & I & $\mathrm{Ba}$ & $\mathrm{M}_{\mathrm{H}}$ \\
\hline \multicolumn{20}{|c|}{ Orculidae } \\
\hline Sdo & Sphyradium doliolum (Brug.) & & & & & & & & & & & & & & + & 6 & $\mathrm{I}$ & Em & $\mathrm{F}_{\mathrm{F}}$ \\
\hline \multicolumn{20}{|c|}{ Enidae } \\
\hline Emo & Ena montana (Drap.) & & & + & + & + & & & & & & & & & + & 25 & II & $\mathrm{Me}$ & $\mathrm{F}_{\mathrm{F}}$ \\
\hline Mob & Merdigera obscura (Müll.) & & & & & & & & & & + & + & & & & 12 & I & Ep & $\mathrm{F}_{\mathrm{F}}$ \\
\hline \multicolumn{20}{|c|}{ Clausiliidae } \\
\hline Cla & Cochlodina laminata (Mont.) & & & & & & & & & & & & & & + & 6 & I & Ep & $\mathrm{F}_{\mathrm{F}}$ \\
\hline Cor & Cochlodina orthostoma (Menke) & & & & & & & & & & & & & & + & 6 & I & $\mathrm{Me}$ & $\mathrm{F}_{\mathrm{F}}$ \\
\hline Rfi & Ruthenica filograna (Rossm.) & & & & & & & & & & & & & & + & 6 & I & Ee & $\mathrm{F}_{\mathrm{F}}$ \\
\hline
\end{tabular}


Table 1. continued

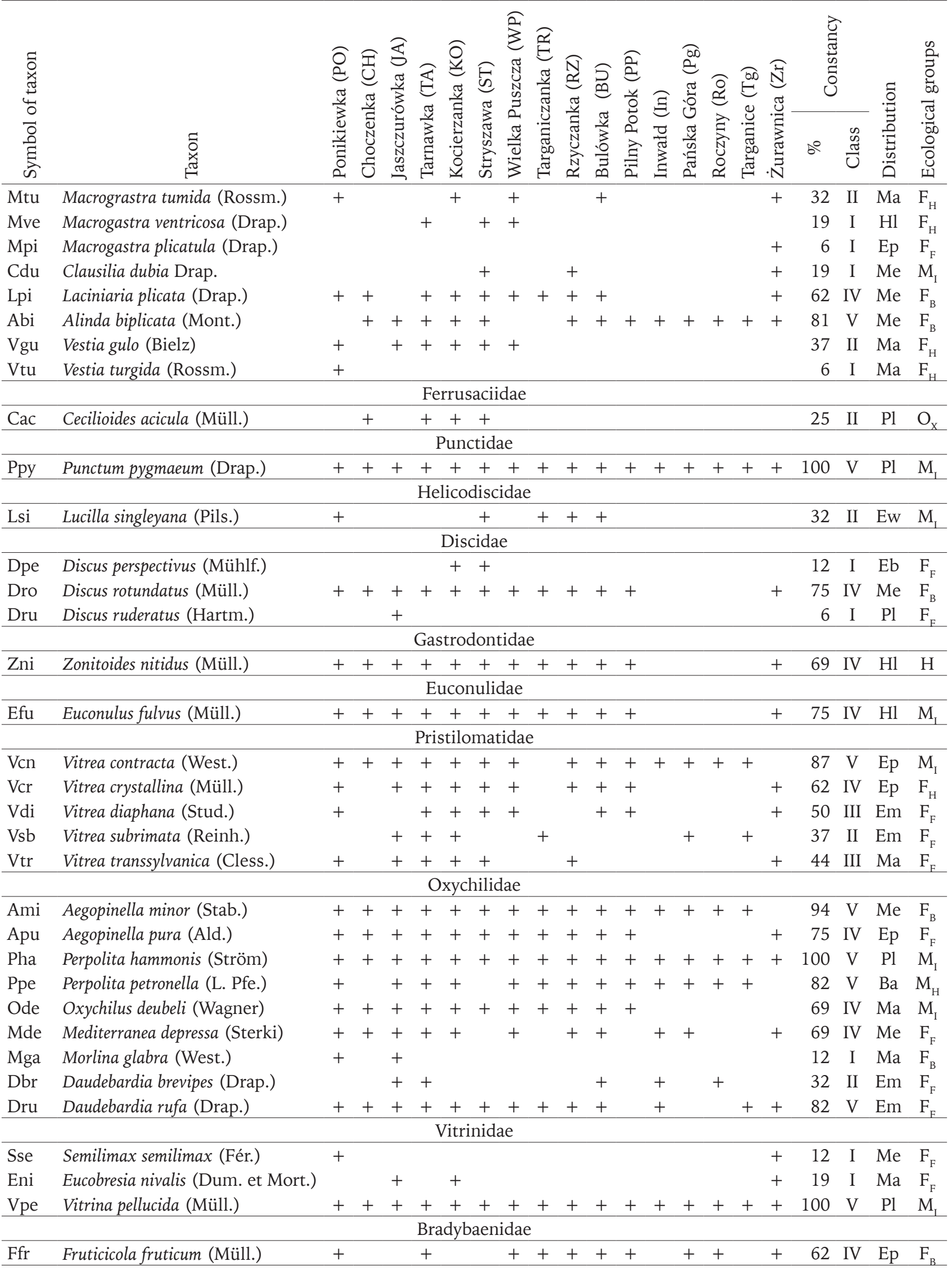


Table 1. continued

\begin{tabular}{|c|c|c|c|c|c|c|c|c|c|c|c|c|c|c|c|c|c|c|c|c|c|c|}
\hline 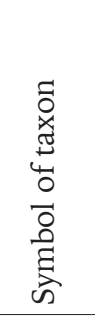 & 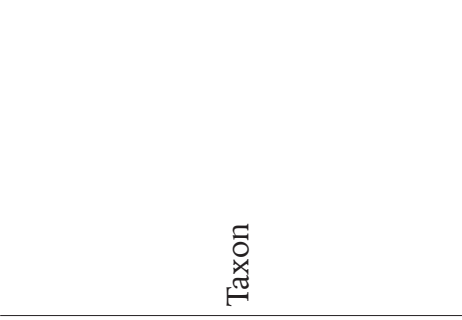 & 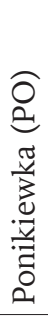 & 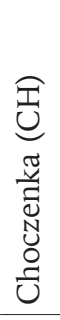 & 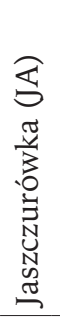 & 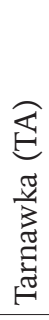 & 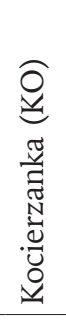 & 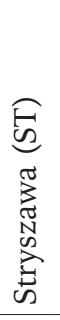 & 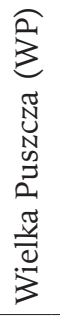 & 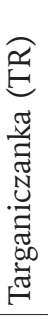 & 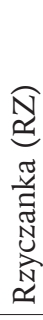 & $\begin{array}{l}0 \\
\frac{\pi}{3} \\
\frac{\pi}{3} \\
0\end{array}$ & & 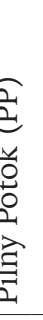 & 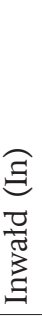 & 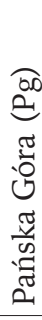 & 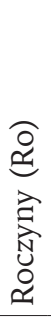 & 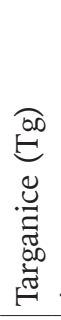 & 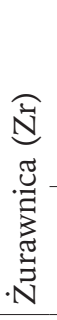 & 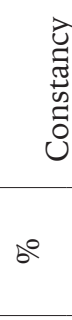 & $\begin{array}{l}\text { D } \\
\underset{\mathbb{U}}{U}\end{array}$ & 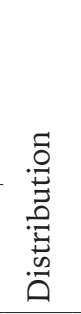 & 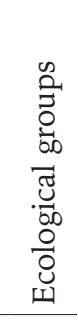 \\
\hline \multicolumn{23}{|c|}{ Hygromiidae } \\
\hline Est & Euomphalia strigella (D) & & & & & & & & & & & & & + & + & & & & 12 & I & Ee & $\mathrm{M}_{\mathrm{D}}$ \\
\hline Tvi & Trochulus villosulus (Rossm.) & + & + & + & + & + & + & + & + & + & + & & + & + & + & + & + & + & 100 & $\mathrm{~V}$ & $\mathrm{Ma}$ & $\mathrm{M}_{\mathrm{H}}$ \\
\hline Thi & Trochulus hispidus (L.) & & + & & + & + & + & + & + & + & + & & + & & & & & & 56 & III & Ep & $\mathrm{M}_{\mathrm{I}}$ \\
\hline Pud & Petasina unidentata (Drap.) & & & + & & & & & & & & & & + & & & + & + & 25 & II & $\mathrm{Ma}$ & $\mathrm{F}_{\mathrm{F}}$ \\
\hline Plu & Plicuteria lubomirskii (Ślós.) & + & + & + & + & + & + & + & + & + & + & & + & + & + & & + & & 87 & $\mathrm{~V}$ & $\mathrm{Ma}$ & $\mathrm{M}_{\mathrm{I}}$ \\
\hline Cun & Candidula unifasciata (Poir.) & & & & & & & & & & & & & + & + & & & & 12 & I & Ew & $\mathrm{O}_{\mathrm{x}}$ \\
\hline Xob & Xerolenta obvia (Menke) & & & & & & & & & & & & & + & + & + & + & & 25 & II & $\mathrm{Eb}$ & $\mathrm{O}_{\mathrm{x}}$ \\
\hline Pbi & Perforatella bidentata (Gmel.) & + & & + & + & & & + & & & & & & & & & & & 25 & II & Ee & $\mathrm{M}_{\mathrm{H}}$ \\
\hline Pru & Pseudotrichia rubiginosa (Rossm.) & & & & + & & & & & & & & & & & & & & 6 & I & Es & $\mathrm{H}$ \\
\hline Min & Monachiodes incarnatus (Müll.) & + & & + & & & + & & & & + & & + & + & + & + & + & + & 62 & IV & $\mathrm{Me}$ & $\mathrm{F}_{\mathrm{B}}$ \\
\hline Mvi & Monachoides vicinus (Rossm.) & + & + & + & + & + & + & + & + & + & + & & + & + & + & + & + & & 94 & $\mathrm{~V}$ & $\mathrm{Ma}$ & $\mathrm{F}_{\mathrm{H}}$ \\
\hline Uurm & Urticicola umbrosus (C. Pfe.) & & + & & + & + & & + & & & & & & + & + & + & & & 44 & III & $\mathrm{Ma}$ & $\mathrm{F}_{\mathrm{H}}$ \\
\hline \multicolumn{23}{|c|}{ Helicidae } \\
\hline Aar & Arianta arbustorum (L.) & + & & + & + & & + & & + & $T$ & + & & & & & & & & 44 & III & $\mathrm{Me}$ & $\mathrm{M}_{\mathrm{H}}$ \\
\hline Ffa & Faustina faustina (Rossm.) & & & & & & & & & & & & & & & & & + & 6 & I & $\mathrm{Ma}$ & $\mathrm{F}_{\mathrm{F}}$ \\
\hline Iis & $\begin{array}{l}\text { Isognomostoma isognomostomos } \\
\text { (Schröt.) }\end{array}$ & + & + & + & + & + & + & + & + & + & + & & + & + & & & & + & 82 & $\mathrm{~V}$ & $\mathrm{Me}$ & $\mathrm{F}_{\mathrm{F}}$ \\
\hline Cne & Cepaea nemoralis (L.) & + & + & & & & & & + & & & & 1 & & & & & & 25 & II & Ew & $\mathrm{F}_{\mathrm{B}}$ \\
\hline Hрo & Helix pomatia ( L.) & + & & + & + & + & + & & & & & & & & & & & & 32 & II & Em & $\mathrm{F}_{\mathrm{B}}$ \\
\hline
\end{tabular}

\section{ECOLOGICAL COMPOSITION OF THE FAUNA}

The component species of the analysed assemblages were classified into nine ecological groups (see Table 1). The greatest proportion (43 species; $55 \%$ of the fauna) is formed by species typical of shaded habitats (ecological groups $F_{F}, F_{B}$ and $F_{H}$ ). Shade-loving species preferring dense forests of low or moderate soil moisture (group $\mathrm{F}_{\mathrm{F}}$ ) include 25 taxa. Some of them occur commonly in the whole massif (e.g. Platyla polita, Aegopinella pura, Isognomostoma isognomostomos), while the others were found only in single localities (e.g. Cochlodina laminata, C. orthostoma, Semilimax semilimax). Species typical of sparse, light-penetrated forests (group $\mathrm{F}_{\mathrm{B}}$ ) (Discus rotundatus, Alinda biplicata) are less numerous (10 taxa). Hygrophilous shade-loving snails (group $\mathrm{F}_{\mathrm{H}}$ ) (8 taxa) are particularly characteristic of valley bottoms overgrown by alders and willows (e.g. Columella edentula, Vestia gulo). The very high proportion of shade-loving taxa is undoubtedly associated with the wide distribution of forest communities within the Beskid Mały area. The limitation of agricultural activities during the last decades resulted in an increase of the degree of afforestation (Fig. 2E, Table 1). Open-country species (groups $\mathrm{O}_{\mathrm{x}}$ and $\mathrm{O}_{\mathrm{O}}$ ) are represented by 8 taxa $(10 \%)$. Vallonia pulchella and $V$. costata are the most numerous while the others are very rare. The small proportion of that group is undoubtedly associated with the low number of open-country habitats. Snails typical of dry, calcareous habitats (Truncatellina cylindrica, Candidula unifasciata and Xerolenta obvia) deserve particular attention. They were recorded in the zone of occurrence of limestones of Andrychów Klippes, particularly in Inwałd and Pańska Góra (AlEXANDrOWICZ 1994). Only empty shells were found in both these locations. It is likely that the species lived there when limestone was quarried in the area. The quarrying favoured retaining of open and dry habitats, providing favourable habitats for the taxa. When the quarrying ceased, the succession in the sites led to extinction of those snails (AlEXANDROWICZ 1994) (Fig. 2E, Table 1). Mesophilous species (groups $\mathrm{M}_{\mathrm{D}}, \mathrm{M}_{\mathrm{r}}$, and $\mathrm{M}_{\mathrm{H}}$ ) constitute the second predominant group (22 species, 28\%). It includes mostly euryoecious taxa of wide distribution (Cochlicopa lubrica, Columella aspera, Euconulus fulvus) (Fig. 2E, Table 1). Hygrophilous species (group H) are few ( 5 species, $6 \%$ of fauna). Their presence is mostly associated with stream valleys, particularly in their lower courses, where the valleys are relatively wide and form floodplains, thus favouring hygrophiles (Fig. 2E, Table 1). 

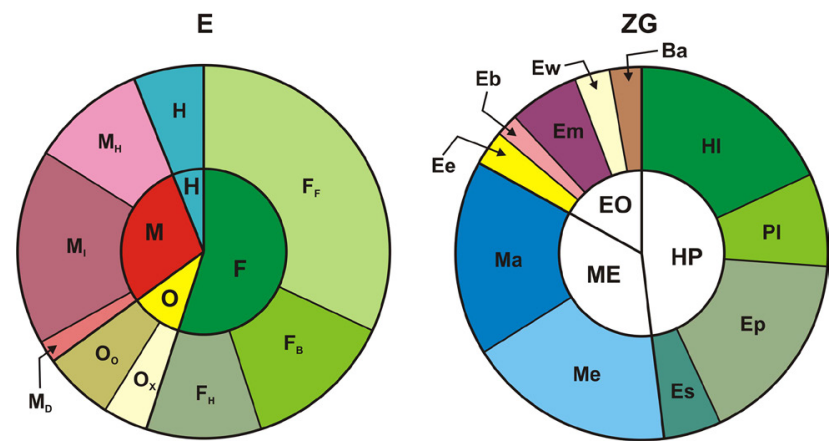

Fig. 2. Ecological (E) and zoogeographical (ZG) structure of the malacofauna. Ecological groups (after LOŽEK 1964, ALEXANDROWICZ \& ALEXANDROWICZ 2011, JUŘIČKOVÁ et al. 2014): $F$ - shade-loving species: $F_{F}$ - shade-loving, forest species, $F_{B}$ - shade-loving species of sparse forests and scrub, $F_{H}$ - shade-loving species of dam habitats; $\mathrm{O}$ - open-country species: $\mathrm{O}_{\mathrm{x}}$ - xerophilous and petrophilous species, $\mathrm{O}_{\mathrm{O}}$ - open-country species; $M$ - mesophilous species: $M_{D}$ - mesophilous species of dry habitats, $M_{I}$ - mesophilous species of moderately wet habitats, $M_{H}$ - mesophilous species of wet habitats; $\mathrm{H}$ - hygrophilous species. Zoogeographical groups (after ALEXANDROWICZ \& ALEXANDROWICZ 2011): $\mathrm{HL}$ - widely distributed species: $\mathrm{Hl}$ - Holarctic species, Pl - Palaearctic species, Ep - European species, Es Euro-Siberian species; ME - Central-European species: $\mathrm{Me}$ - Central-European lowland and upland species, $\mathrm{Ma}$ - Central-European mountain species; EO - species of narrow distribution: Ee - East-European species, Eb - Pontic and Balkan species, Em - Mediterranean species, Ew - West-European species, Ba - Boreal-Alpine species

\section{ZOOGEOGRAPHICAL COMPOSITION OF THE FAUNA}

Three principal zoogeographical groups are represented in the analysed assemblage. The first includes widely distributed taxa (group HP): Holarctic $(\mathrm{Hl})$, Palaearctic (Pl), European (Ep), and EuroSiberian (Es) species. The group comprises 34 taxa ( $44 \%$ of the fauna) and includes common species inhabiting almost the whole of the Beskid Mały range (Fig. 2ZG, Table 1). An essential role is played by Central-European (ME) forms (27 taxa, 35\%). The proportion of lowland-upland snails $(\mathrm{Me})$ and that of mountain species (Ma) is almost the same (Fig. 2ZG, Table 1). Conclusions from the malacological studies in other ranges of the Beskidy Zachodnie Mts (Babia Góra, Gorce) (ALEXANDrowicz 2003, 2011, KASZUBA \& STWORZEWICZ 2018) are similar. Evident prevalence of mountain taxa is marked only in the Tatra Mts (DYDUCH-FALNIOWSKA 1991). The snails of limited distribution range (EO) complement the assemblage. This group is not very numerous (17 species, $21 \%$ ), with Mediterranean (Em) taxa playing the principal role. The small number of Boreal-Alpine $(\mathrm{Ba})$ forms is the point of interest here (Fig. 2ZG, Table 1).

\section{DISTRIBUTION OF MALACOFAUNA IN THE BESKID MAŁY RANGE}

The studies covered considerable areas in the eastern part of the Beskid Mały. Owing to the high number of localities, it was possible to determine the areas of occurrence for particular species within this mountain range. Constancy analysis (C) made it possible to indicate common species, as well as rare taxa whose occurrence is limited to its parts, and sometimes only to single, isolated sites. Among the 78 species found in the Beskid Mały, 34 taxa (44\% of the assemblage) occurred in at least $60 \%$ localities (constancy classes V and IV) (Fig. 3, Table 1). These are the taxa whose distribution includes practically the whole area of the Beskid Mały. Twenty two species were found only in single localities (constancy class I) (Fig. 3, Table 1). Distribution of the remaining classes was limited to some parts of the massif (constancy classes II and III) (Fig. 3, Table 1). Approximated distribution of particular taxa of snails in the Beskid Mały range is presented in Figs 4-6.
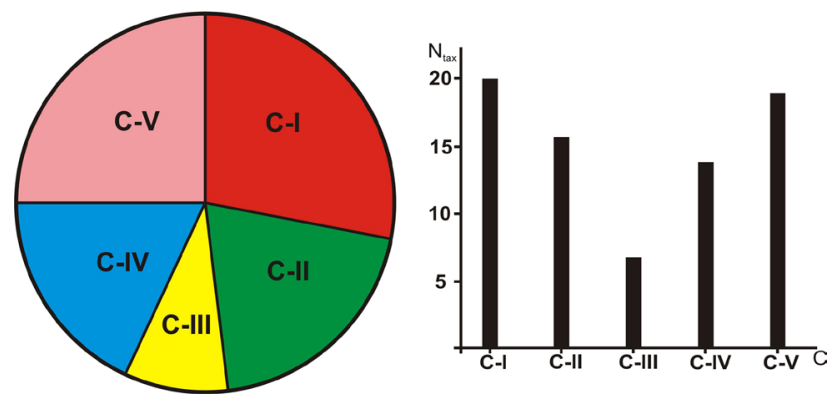

Fig. 3. Constancy structure of the malacofauna. C-I C-V (constancy classes, after ALEXANDROWICZ \& ALEXANDROWICZ 2011), $\mathrm{N}_{\text {tax }}$ - number of taxa

\section{DIVERSITY OF MALACOFAUNA IN THE BESKID MAEY RANGE}

The dendrogram separated four types of fauna in the Beskid Mały range, of different species composition and ecological and zoogeographical structure.

Type A, with its 66 species, is the richest. It is characterised by a great proportion of shade-loving species, particularly those of compact forest communities. They are accompanied by mesophilous forms. Open-country species and hygrophilous snails constitute complementary elements. Widely distributed taxa (HP) and Central-European species (ME) predominate. The great proportion of mountain species (Ma) is noteworthy. The fauna inhabits the main ridge of the Beskid Mały, namely the Leskowiec-Łamana Skała ridge. It is an area overgrown by dense mixed forests or beech forests with a very small proportion of open habitats (Figs 7 and 8). 


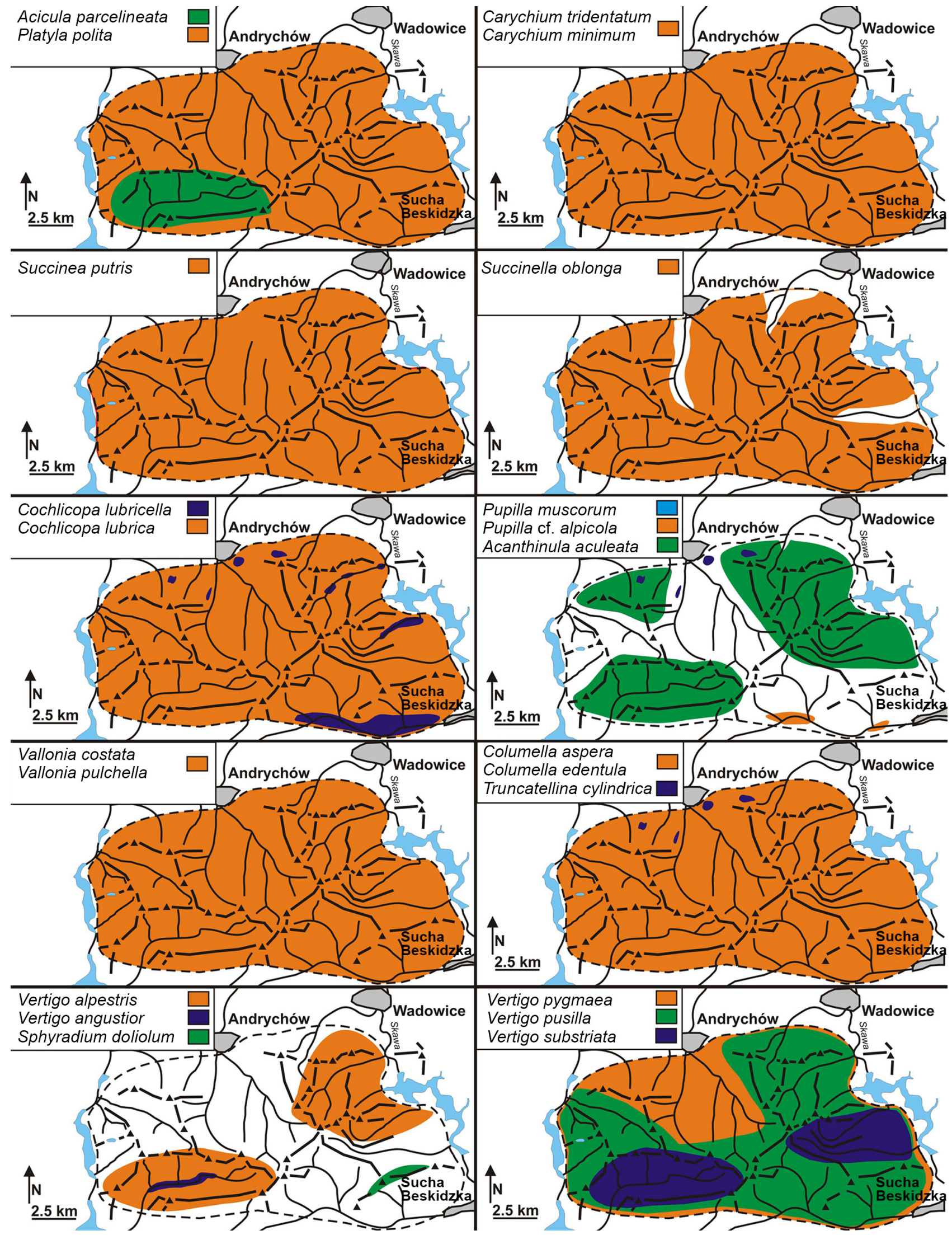

Fig. 4. Approximated distribution of species in the Beskid Mały (part I). For explanations see Fig. 1 


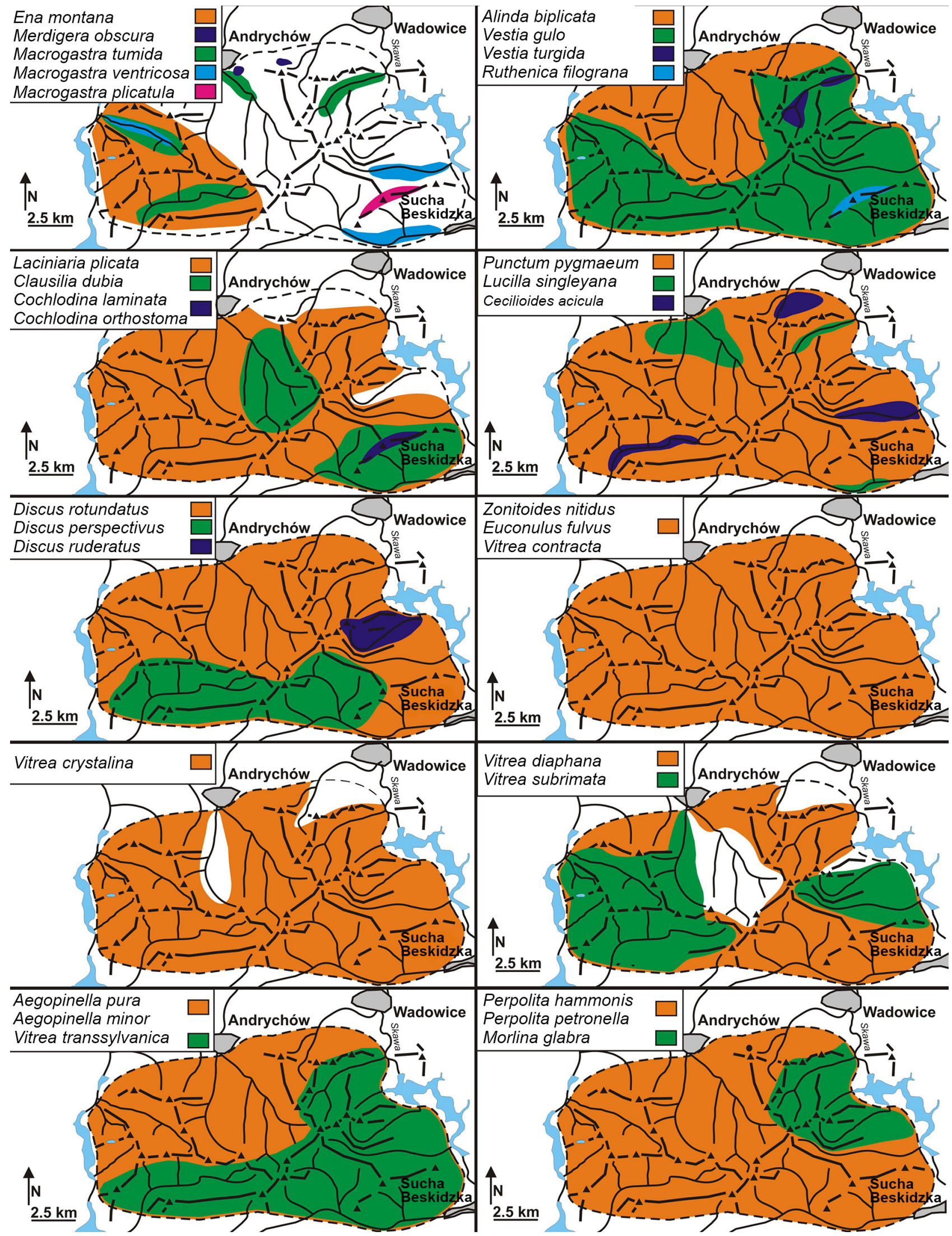

Fig. 5. Approximated distribution of species in the Beskid Mały (part II). For explanations see Fig. 1 


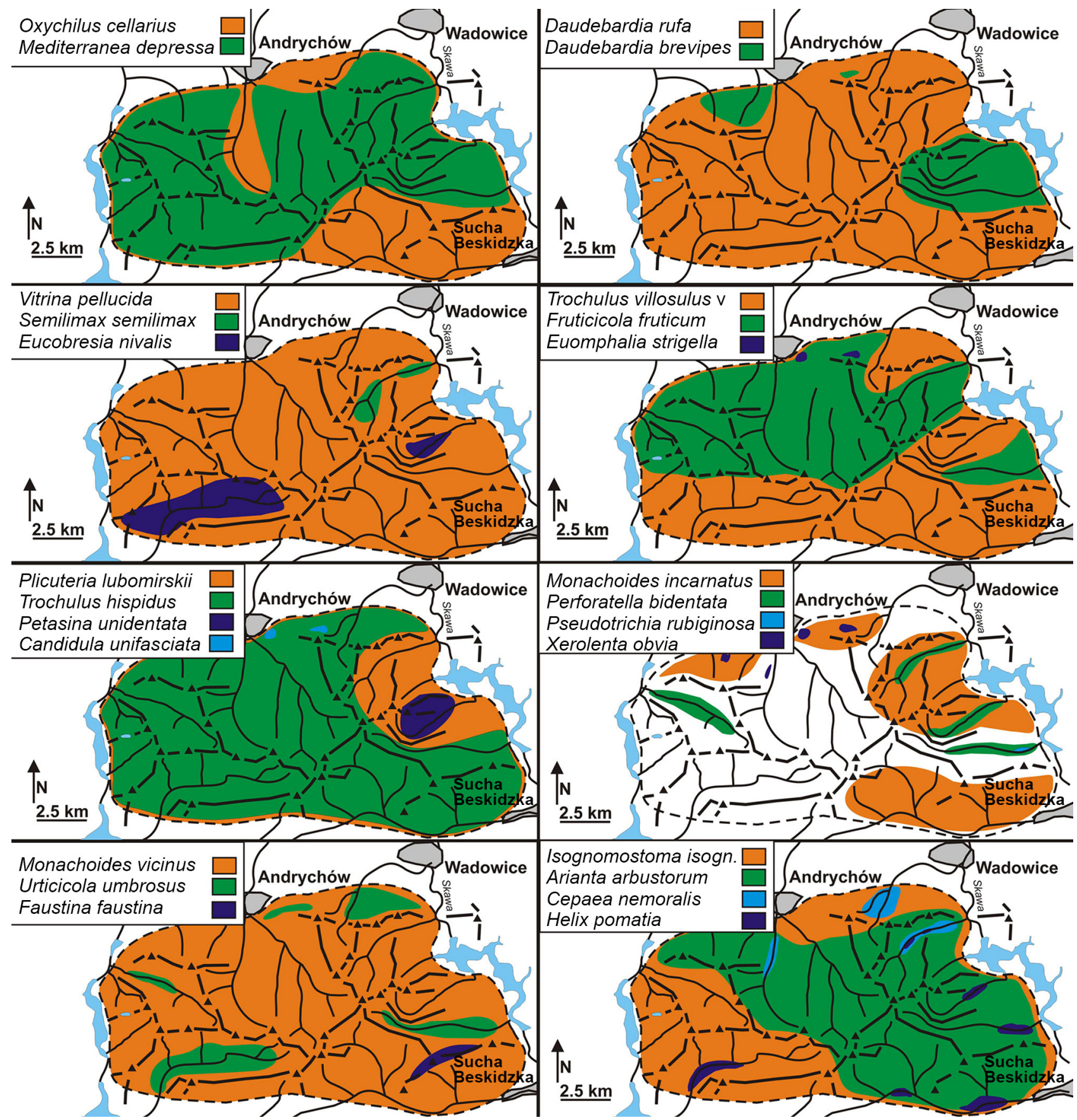

Fig. 6. Approximated distribution of species in the Beskid Mały (part III). For explanations see Fig. 1

Type B includes 52 species. It is characterised by a smaller proportion of shade-loving taxa compared to type A. Species typical of scrub and sparse forests are particularly numerous. The frequency of open-country species and mesophilous forms is also higher. The proportion of Central-European taxa (ME), particularly the mountain ones, is limited at the expense of widely distributed species (HP). The fauna occurs in the northern part of the Beskid Mały range (Figs 7 and 8).

Type C shows the smallest species diversity (34 taxa), however, some of its component species do not occur in the other types of fauna. They include
Pupilla muscorum, Merdigera obscura, Euomphalia strigella, Candidula unifasciata and Xerolenta obvia. The first two are relatively abundant, particularly Merdigera obscura, and the remaining three were found only as empty shells, often severely damaged (ALEXANDROwICZ 1994). Additionally, these three species are xerophilous and calciphilous forms preferring open and even xerothermic habitats. They lived there at the time when limestone was quarried. The quarries were deforested and dry habitats on calcareous substrate occurred. After the mining ceased the quarries were overgrown, resulting in disappearance of open habitats and extinction of the species in ques- 


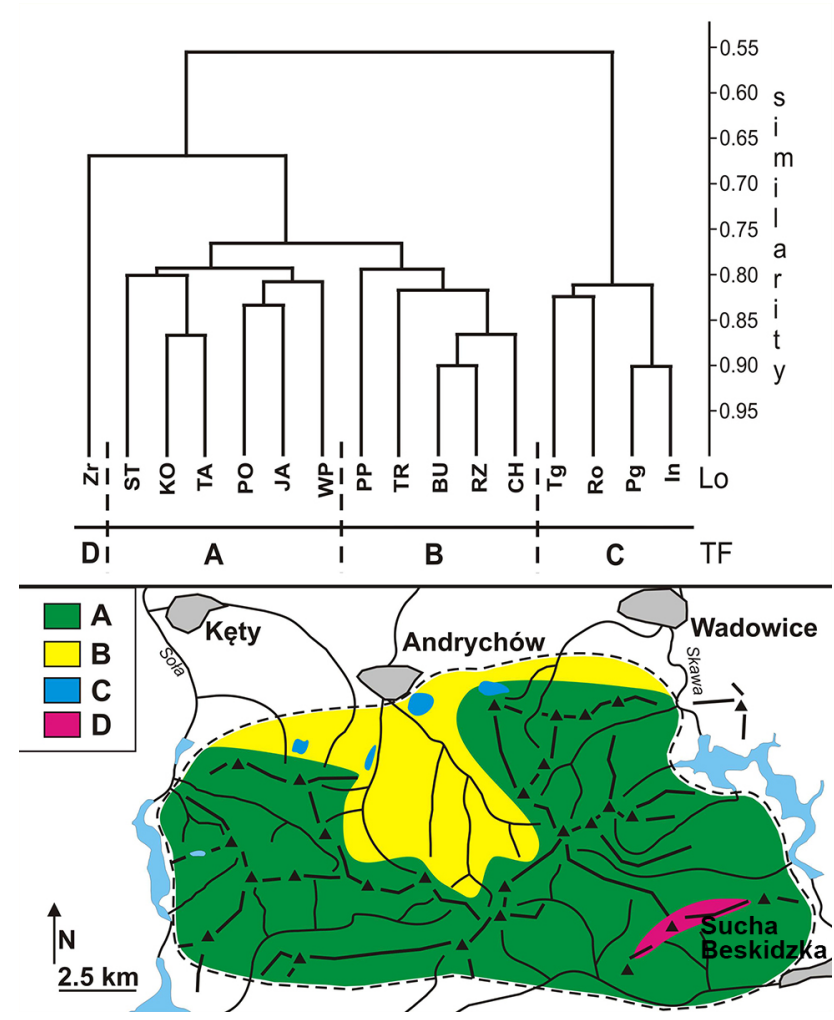

Fig. 7. Diversity of the malacofauna in of the Beskid Mały. A-D - types of fauna described in the text. Other explanations as in Fig. 1
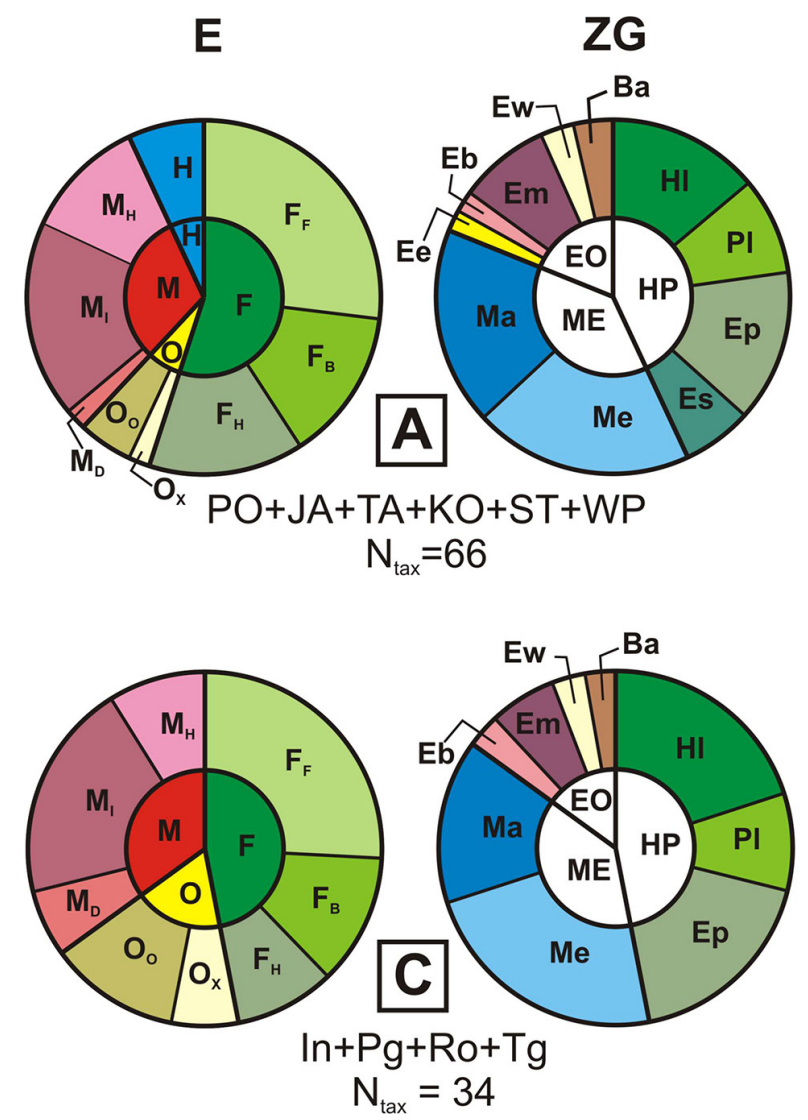

tion (ALEXANDROWICZ 1994). The presented type of fauna is also characterised by a great proportion of open-country snails and by complete absence of hygrophilous taxa. The great proportion of CentralEuropean upland species is noteworthy. The described fauna occurs within the limestone outcrops forming Andrychów Klippes in the northern part of the range (Figs 7 and 8).

Type D is a diverse fauna of 39 species. Its characteristic features include a very high number of shade-loving species. Mesophilous taxa and open-country snails supplement the fauna, but hygrophilous forms are absent. Some components of the fauna in question were not recorded in the other faunas described above. Those are mostly shade-loving snails: Sphyradium doliolum, Cochlodina laminata, C. orthostoma, Ruthenica filograna, Macrogastra plicatula and Faustina faustina. The zoogeographical composition is characterised by a high number of European and Central European upland species, as well as a relatively high proportion of Mediterranean taxa. The described fauna occurs in the Żurawnica range, particularly in the zone of outcrops of slightly calcareous Ciężkowice sandstones of the Magura unit, which form this range of small rock forms (ALEXANDROWICZ \& ALEXANDROWICZ 2006) (Figs 7 and 8).
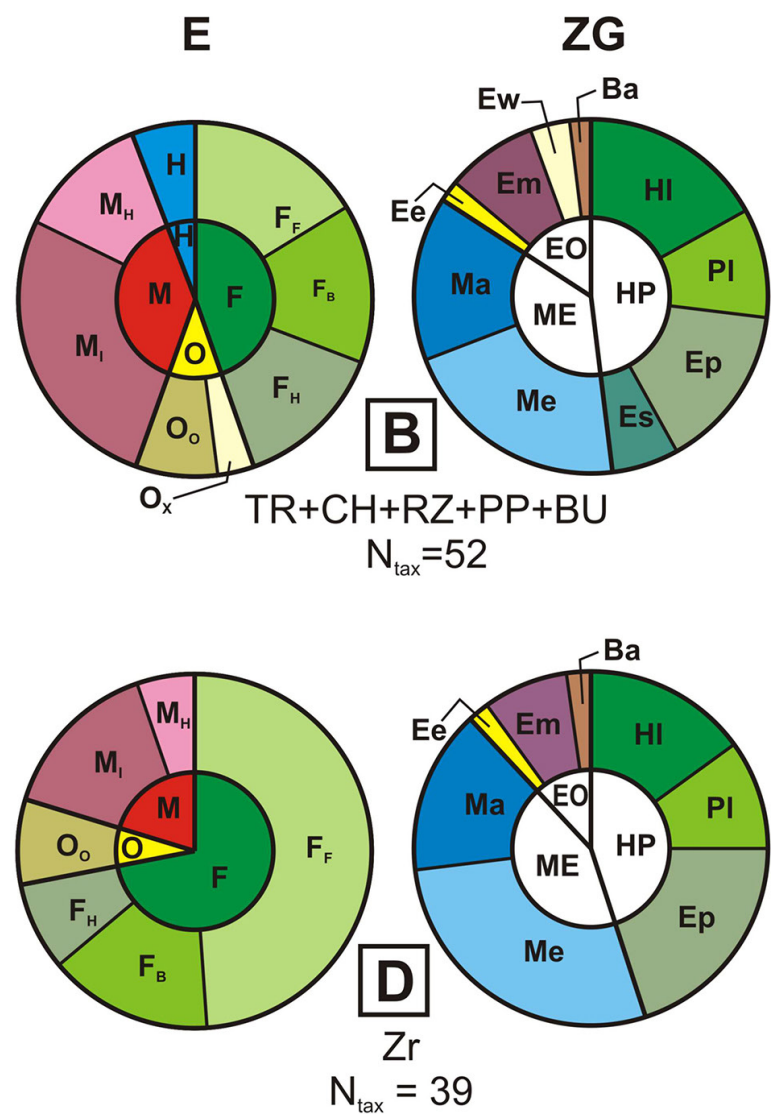

Fig. 8. Ecological (E) and zoogeographical (ZG) diversity of the malacofauna of the Beskid Mały. A-D - types of fauna described in text, $\mathrm{N}_{\operatorname{tax}}$ - total number of species in particular types of fauna. Other explanations as in Figs 1 and 2 


\section{SUMMARY AND CONCLUSIONS}

Compared to the other ranges in the Polish Carpathians, the malacofauna of the Beskid Mały is rich and diverse. It includes 78 species of terrestrial snails, a number comparable to those recorded for the Babia Góra massif - 78 species (AlEXANDrowiCZ 2003, 2011), Gorce - 53 species (KASZUBA \& STWORZEWICZ 2018), or Tatra Mts 70 species (DYDUCH-FALNIOWSKA 1991). Richer and more diverse mollusc assemblages are known from the Pieniny Mts - 84 species (URBAŃSKI 1939, STWORZEWICZ 2000), the Podhale Basin - 81 species (AlEXANDROWICZ 1997b, 2000b, c, 2013b) and Bieszczady and Beskid Niski Mts - 90 species (STWORZEWICZ \& PAWŁOWSKI 2000, SULIKOWSKADrOZD 2002, SULIKOWSKA-DROZD \& HORSÁK 2007). Such a great diversity of the malacofauna in the Beskid Mały stems from a number of factors. The geological structure of the bedrock should be considered a fundamental factor. It affects the chemical composition of the soil, the predominant type of vegetation formations and land relief and, as a further consequence, the suitability of the areas for the purpose of human settlement and agriculture. The northern part of the area is situated in the zone made of shale complexes of the Sub-Silesian and Silesian units. The area is characterised by gentle relief and has been deforested and used as farmland for many years. The expansion of scrub and forest communities has taken place only during the last few decades. As a consequence, mesophilous species and forms typical of sparse forests predominate (Type B fauna). The major part of the Beskid Mały range (the Leskowiec-Łamana Skała range) is built of sandstone links of the Silesian unit with a small content of carbonates. The remarkable resistance of those rocks to denudation leads to formation of mountain ranges with steep slopes which are cut by narrow and deep valleys. These areas are overgrown by compact forests with a high proportion of Carpathian beech forest. The mollusc fauna

\section{REFERENCES}

AlEXANDROWICZ S. W. 1995. Ruins of Carpathian castles as refuges of land snails. Rocznik Ochrona Przyrody 52: 2-18.

ALEXANDROWICZ S. W. 1997a. Death assemblages of molluscs in flood deposits of the Muszynka River (Polish Carpathians). Studia Geomorphologica CarpathoBalcanica 31: 111-127.

ALEXANDROWICZ S. W. 2000a. Mollusc thanatocenoses of the Vistula River in the southern part of the Cracow Upland. Folia Malacologica 8: 171-179. https://doi. org/10.12657/folmal.008.012 living there shows great species diversity and predominance of forms typical of compact forests (Type A fauna). A unique geological phenomenon in the Beskid Mały range is the occurrence of limestones (Andrychów Klippes). The great carbonate content favours the diversity of malacofauna. Despite these favourable conditions, the fauna identified there is characterised by relatively small species diversity. Additionally, it includes calciphilous and xerophilous taxa (Euomphalia strigella, Candidula unifasciata and Xerolenta obvia), whose populations developed there during the limestone quarrying period. At present, they can be considered extinct in the area (Type C fauna). The Żurawnica range, which is the southernmost part of the Beskid Mały range, is inhabited by the assemblage with predominance of forest-dwellers (Type D fauna).

In the Beskid Mały range several rare and protected snail species were found. Those include the Carpathian endemic and sub-endemic species which are under strict or partial species protection, being listed in the Red Book of Threatened Species in Poland (WIKTOR \& RIEDEL 1992) and in the Carpathian List of Endangered Species (WITKOWSKI et al. 2003), as well as the taxa listed in Annex II to the EU Habitats Directive. Also present are some snails not included in the above lists but rare in the Carpathians or with poorly known distribution. This group includes almost 30 taxa. Detailed information on their occurrence in the Beskid Mały and their protection status were described in another paper (ALEXANDROWICZ 2019).

\section{ACKNOWLEDGEMENTS}

This study has been sponsored by AGH University of Science and Technology via the University grant No 11.11.140.005.

AleXANDrowicz S. W., AleXANDrowicz W. P. 2011. Analiza malakologiczna. Metody badań i interpretacji. Rozprawy Wydziału Przyrodniczego PAU 3: 3-302.

AleXANDrowicz S. W., AleXANDrowiCZ Z. 2006. Rocky Edge and malacocenoses of the Żurawnica ridge (Polish Western Carpathians). Ekologia (Bratislava) 25: 151165.

AlEXANDROWICZ W. P. 1994. Współczesna i subfosylna malakofauna kamieniołomu na Pańskiej Górze w Andrychowie. Rocznik. Ochrona Przyrody 51: 147156. 
ALEXANDROWICZ W. P. 1997b. Malakofauna osadów czwartorzędowych i zmiany środowiska naturalnego Podhala w młodszym vistulianie i holocenie. Folia Quaternaria 68: 7-132.

Alexandrowicz W. P. 2000b. Tanatocenozy muszlowe przy wodospadzie Kacwińskiego Potoku na Podhalu. Chrońmy Przyrodę Ojczystą 56: 28-43.

AlEXANDROWICZ W. P. 2000c. Tanatocenozy muszlowe $z$ osadów powodziowych w dolinach potoków na Podhalu. Geologia Kwartalnik AGH 26: 449-467.

AlEXANDROWICZ W. P. 2002. Mollusc thanatocoenoses in flood deposits of the Beskid Mały range and foothills (Western Carpathians, Poland). Bulletin of the Polish Academy of Sciences, Earth Sciences 50: 67-80.

AleXANDrowicz W. P. 2003. Ślimaki (Mollusca; Gastropoda) i małże (Mollusca; Bivalvia) masywu Babiej Góry. In: WoŁoszYN B. W., WoŁoszYN D., Celary W. (eds). Monografia Fauny Babiej Góry. Publikacje Komitetu Ochrony Przyrody PAN: 69-97.

AlEXANDROwiCz W. P. 2011. Ślimaki lądowe Babiogórskiego Parku Narodowego. Chrońmy Przyrodę Ojczystą 67: 39-54.

ALEXANDROWICZ W. P. 2013a. The malacofauna of the castle ruins in Melsztyn near Tarnów (Rożnów Foothills, southern Poland). Folia Malacologica 21: 9-18. https:// doi.org/10.12657/folmal.021.002

AlEXANDROWICZ W. P. 2013b. Molluscan communities in Late Holocene fluvial deposits as an indicator of human activity: a study in Podhale Basin in Southern Poland. Ekologia (Bratislava) 32: 111-125. https://doi org/10.2478/eko-2013-0010

AlEXANDROWICZ W. P. 2019. Rzadkie i chronione ślimaki lądowe Beskidu Małego. Chrońmy Przyrodę Ojczystą 75: 83-97.

BĄKOWSKI J. 1883. Mięczaki tatrzańskie. Kosmos 8: 13-17.

BĄKOWSKI J. 1884. Mięczaki galicyjskie. Kosmos 9: 190197, 275-283, 376-391, 477-490, 604-611, 680-697, 761-798.

CIESZKOWSKI M., GolONKA J., WAŚKOWSKA-OliWA A., CHRUstek M. 2006. Budowa geologiczna rejonu Sucha Beskidzka - Świnna Poręba (polskie Karpaty fliszowe). Geologia Kwartalnik AGH 32: 155-201.

ČEJKA T., HorsáK M., NÉMETHOVÁ D. 2008. The composition and richness of Danubian floodplain forest land snail faunas in relation to forest type and flood frequency. Journal of Molluscan Studies 74: 37-45. https://doi. org/10.1093/mollus/eym041

ČILIAK M., ČEJKA T., ŠTEFFEK J. 2015. Molluscan diversity in stream driftwood: relation to land use and river section. Polish Journal of Ecology 63: 124-134. https:// doi.org/10.3161/15052249PJE2015.63.1.011

DYDUCH-FALNIOWSKA A. 1991. The gastropods of the Polish Tatra Mountains. Studia Naturae seria A 38: 5-111.

DzIĘCZKOWsKI A. 1971. Badania ilościowe mięczaków buczyn południowo-wschodniej Polski. Sprawozdania PTPN 83: 258-260.

DZIĘCZKOWSKI A. 1972. Badania ilościowe mięczaków buczyn południowo-wschodniej Polski. Studium ekolo- giczno-faunistyczne. Prace Komisji Biologicznej PTPN 35: 243-332.

DZIĘCZKOWSKI A. 1988. Zespoły ślimaków (Gastropoda) zbiorowisk leśnych Polski. Prace Komisji Biologicznej PTPN 68: 1-117.

GASIŃSKI A. 1998. Campanian-Maastrichtian paleoecology and paleobiogeography of the Andrychów Klippes, Outer Carpathians, Poland. Wydawnictwo UJ, Rozprawy Habilitacyjne 333: 1-90.

HAMmer Ø., HARPER D. A. T., RYAN P. D. 2001. Past: paleontological statistics software package for education and data analysis. Palaeontologica Electronica 4: 1-9.

HorsÁK M., JUŘIČKOVÁ L., PICKA J. 2013. Měkkýši České a Slovenské republiky, Molluscs of the Czech and Slovak Republics. Nakladatelstvi Kabourek, Zlín.

Ilg C., Foeckler F., Deichner O., Henle K. 2009. Extreme flood events favour floodplain mollusc diversity. Hydrobiologia 621: 63-73. https://doi.org/10.1007/ s10750-008-9632-5

JUŘiČKOVÁ L., HORSÁK M., HORÁČKOVÁ J., LOŽEK V. 2014. Ecological groups of snails - use and perspectives. European Malacological Congress, Cambridge, UK. Available online at http://mollusca.sav.sk/malacology/ Jurickova/2014-ecological-groups-poster.pdf.

KASZUBA M., STWORZEWICZ E. 2018. Malakocenozy Gorczańskiego Parku Narodowego ze szczególnym uwzględnieniem zespołu buczyny karpackiej. Chrońmy Przyrodę Ojczystą 74: 103-116.

Kerney M. P., Cameron R. A. D., Jungbluth J. H. 1983. Die Landschnecken Nord- und Mitteleuropas. Verlag Paul Parey, Hamburg-Berlin.

KOTULA B. 1884. O pionowém rozsiedleniu ślimaków tatrzańskich. Sprawozdanie Komisji Fizjograficznej 18: 139-203.

KSIĄŻKIEWICZ M. 1932. Budowa geologiczna brzeżnych Beskidów Wadowickich i ich stosunek do przedmurza. Rocznik Polskiego Towarzystwa Geologicznego 8: 49-93.

KSIĄŻKIEWICZ M. 1951. Objaśnienia do arkusza Wadowice. Wydawnictwo PIG, Warszawa.

LOŽEK V. 1964. Quartärmollusken der Tschechoslowakei. Rozpravy Ustředniho Ustavu Geologického 31: 1-374.

MORISITA M. 1959. Measuring of interspecific association and similarity between communities. Memories of the Faculty of Sciences, Kyushu University E 3: 65-80.

MYŠÁK J., HorsÁK M. 2011. Floodplain corridor and slope effects on land mollusc distribution patterns in a riverine valley. Acta Oecologica 37: 146-154. https://doi. org/10.1016/j.actao.2011.01.012

PAUL Z., RYŁKO W., TOMAŚ A. 1996. Zarys budowy geologicznej zachodniej części Karpat polskich (bez utworów czwartorzędowych). Przegląd Geologiczny 44: 469-476.

RIEDEL A. 1988. Ślimaki lądowe. Gastropoda terrestria. Katalog Fauny Polski 36. PWN, Warszawa.

STOBIECKI S. 1880. Spis mięczaków zebranych na Babiéj Górze w r. 1879..., oznaczonych przez p. J. Bąkowskiego. Sprawozdanie Komisji Fizjograficznej 14: 77-78. 
STOBIECKI S. 1883. Do fauny Babiéj Góry. Sprawozdanie z wycieczek entomologicznych na Babią Górę w latach 1879 i 1880. Sprawozdanie Komisji Fizjograficznej 17: $1-84$.

STwORZEWICz E. 2000. Mięczaki (Mollusca). Flora i Fauna Pienin. Monografie Pienińskie 1: 87-91.

STWORZEWICZ E., PAWŁOWSKI J. 2000. Mięczaki (Mollusca) Bieszczadów Zachodnich. Roczniki Bieszczadzkie 7: 255-260.

SulikOWSKA-DROZD A. 2002. Uzupełnienie listy ślimaków lądowych Bieszczadów Zachodnich. Roczniki Bieszczadzkie 10: 343-352.

SULIKOWSKA-DROZD A., HorsÁK M. 2007. Woodland mollusc communities along environmental gradients in the East Carpathians. Biologia (Bratislava) 62: 201-209. https://doi.org/10.2478/s11756-007-0030-7

URBAŃSKI J. 1932. Die Molluskenfauna der Babia Góra (Westkarpaten). Archiv für Molluskenkunde 24: 117136.

URBAŃSKI J. 1939. Mięczaki Pienin ze szczególnym uwzględnieniem polskiej części Parku Narodowego. Prace komisji Matematyczno-Przyrodniczej PTPN, B 9: 265-505.
URBAŃSKI J. 1962. Mięczaki. In: Tatrzański Park Narodowy. Wydawnictwo Zakładu Ochrony Przyrody PAN Kraków, pp. 473-484.

WELTER-SCHULTES F. 2012. European non-marine molluscs, a guide for species identification. Planet Poster Editions, Göttingen.

WASMUND E. 1926. Biocoenose und Thanatocoenose. Archiv für Hydrobiologie 17: 1-116.

WIKTOR A. 2004. Ślimaki lądowe Polski. Wydawnictwo Mantis, Olsztyn.

WIKTOR A., RiEdEL A. 1992. Ślimaki lądowe. Gastropoda terrestria. In: GŁOWACIŃsKI Z. (ed.). Czerwona lista zwierząt ginących i zagrożonych w Polsce. Zakład Ochrony Przyrody PAN, pp. 31-38.

WiTKOWSKI J., KRÓL W., SOLARZ W. 2003. Carpathian list of endangered species. Europress Kraków.

Received: April 18th, 2019

Revised: September 24th, 2019

Accepted: September 28th, 2019

Published on-line: October 30th, 2019 